\title{
De-noising of eddy current array signal based on empirical mode decomposition threshold
}

\author{
Bonan Yang ${ }^{a}$, Zhijun Zhang ${ }^{b}$, Jinqiang $\mathrm{Du}^{\mathrm{c}}$ and Yubing Wang ${ }^{\mathrm{d}}$ \\ Aeronautics and Astronautics Engineering College, Air Force Engineering University, Xi'an 710038, \\ China; \\ asuming_yangbonan@163.com, ${ }^{\mathrm{b}}$ Zhangzhijun_1960@163.com,

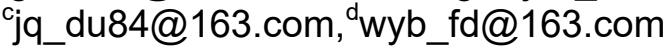

Keywords: eddy current array signal, empirical mode decomposition(EMD), wavelet threshold de-noising.

\begin{abstract}
A method based on the empirical mode decomposition (EMD) threshold de-noising algorithm is proposed since eddy current array signal is mixed with more high frequency noise. This algorithm consists of four steps: (i) The signal is decomposed into IMFs by EMD, (ii) the signal is recombined based on energy reduction of the first IMF, and then the recombined signal is decomposed by EMD, (iii) IMFs of much noise according to the noise statistics are de-noised by threshold de-noising, (vi) de-noised IMFs and non-processed IMFs are reconstructed into the de-noised signal. Experimental and simulation results show that this new method can get higher SNR, reduce the RMSE, and be more effective to recover the original signal.
\end{abstract}

\section{Introduction}

Eddy current array detection is an important branch of Nondestructive Testing(NDT). Eddy current array signal in the generation, propagation and reception of the process produces noise, and the signal has been interfered by noise[1]. In order to carry on the further analysis and processing to the original signal, it is necessary to take effective ways to eliminate the noise of the original signal[2]. Empirical mode decomposition (EMD) is a new algorithm to deal with non-stationary signals, which is proposed by E.Huang Norden in 1998[3]. Compared with the wavelet transform, empirical mode decomposition is based on the characteristic time scale of the signal itself, which can be decomposed into the intrinsic mode functions(IMF) of different scales. When the signal is processed by EMD threshold de-noising, there are some problems like distortion. A new EMD threshold de-noising algorithm based on the characteristics of noise is proposed in order to overcome the deficiency of EMD threshold de-noising. Experiment shows that the proposed algorithm is better than the original method and can restore the original signal.

\section{The basic principle of EMD}

The basic process of EMD decomposition is as follows[4] :

1)First automatically calculate the signal $x(t)$ all the maxima and minima, fitting the signal on the $u(t)$ and the lower envelope $v(t)$, and the average value of the envelope $m(t)$ :

$$
\begin{aligned}
& m(t)=\frac{1}{2}[u(t)+v(t)] \\
& \text { 2) set } \\
& h_{1}(t)=x(t)-m(t)
\end{aligned}
$$

To determine whether $h_{1}(t)$ to meet the two conditions of the intrinsic function, $h_{1}(t)$ for the first intrinsic function; otherwise, repeat steps(1),(2), until $h_{1}(t)$ to meet the conditions, denoted as $c_{1}(t)=$ $h_{1}(t)$.

3) Remove $c_{1}(t)$ from $x(t)$, get a new signal that is waiting to be processed:

$$
r_{1}(t)=x(t)-c_{1}(t)
$$


4) $r_{1}(t)$ as the original sequence, repeat steps (1), (2), (3), to get the remaining signal components, denoted as $c_{1}(t), c_{2}(t), \ldots, c_{\mathrm{n}}(t)$. if the signal is an IMF which is check by

$$
S_{d}=\sum_{t=0}^{T} \frac{\left|h_{k-1}(t)-h_{k}(t)\right|^{2}}{h_{k}^{2}(t)}
$$

The value of $S_{d}$ is generally set between 0.2 and 0.3 .

The signal decomposition form is as follows:

$$
x(t)=\sum_{i=1}^{n} c_{i}(t)+r_{n}(t)
$$

\section{Improved threshold de-noising algorithm based on EMD}

One dimensional noise signal[5]:

$x(t)=f(t)+e(t), t=0,1, \cdots, n-1$ noise.

$x(t)$ is signal containing noise, $f(t)$ is low frequency useful signal, and $e(t)$ is high frequency

After EMD decomposition, $c_{1}(t)$ is similar to a low signal to noise ratio zero mean signal. According to the characteristics of this signal[6], $c_{1}(t)$ can be processed to reduce the interference of noise first:

$c_{1}^{\prime}(t)$ is obtained by randomly changing the position of each sequence in $c_{1}(t)$. Because the amplitude of each sequence is not changed, the energy of the signal is not changed. $c_{L}^{\prime}(t)$ is obtained through the $\mathrm{L}$ times to do $c_{1}(t)$ sequence of random transformation, accumulation, and then seek the average. With the increase of $\mathrm{L}$, the noise energy of $W_{c}^{\prime}$ becomed smaller and smaller:

$$
c_{L}^{\prime}(t)=\frac{\sum_{i=1}^{L} c_{i}(t)}{L+1}
$$

After the processing of $c_{L}^{\prime}(t)$, its noise energy has been quite low. The new signal $x^{\prime}(t)$ is obtained with adding $c_{L}^{\prime}(t)$ to the IMFs $c_{2}(t)$ to $c_{n}(t)$. New IMFs are obtained by EMD of $x^{\prime}(t)$, in which the low order IMFs contains more noise; higher order IMFs contains the useful signal, less noise. In order to determine the boundary points of the noise IMFs and the useful IMFs, a method based on the statistical characteristics of Gauss white noise was used as follow:

Calculate the energy density $E_{i}$ and the average period $\bar{T}_{i}$ of each IMF, and obtain the product:

$P_{i}=E_{i} \cdot \overline{T_{i}}, i=1,2, \cdots, n$

Set

$R_{i}=P_{i} / P_{i-1}, i=1,2, \cdots, n$

After Gauss white noise is decomposed by EMD, $R_{i}$ of each order IMF is constant. When the useful signal occurs, $R_{i}$ is a local minimum. When $R_{i} \geq 2$, the useful signal in the $k$-th IMF is dominant, and $k$ is the dividing point of the useful signal and the noise signal.

The steps of threshold de-noising algorithm based on EMD are as follows:

Step 1) Get $n$ IMFs by EMD of $x(t)$.

Step 2) Get $c_{L}^{\prime}(t)$ by producing $c_{1}(t)$, set $s_{1}(t)=\sum_{i=2}^{n} c_{i}(t)$, then $x^{\prime}(t)=c_{L}^{\prime}(t)+s_{1}(t)$.

Step 3) Get new IMFs $c_{1}^{\prime}(t)$ to $c_{n}^{\prime}(t)$ by EMD of $x^{\prime}(t)$, and determine the dividing point $k$.

Step 4) Obtain $\sum_{i=1}^{k} c_{i}^{\prime \prime}(t)$ through wavelet threshold de-noising of the noise IMFs. 
Step 5) Reconstruct $x^{\prime \prime}(t)=\sum_{i=1}^{k} c_{i}^{\prime \prime}(t)+\sum_{j=k+1}^{n} c_{j}{ }^{\prime}(t)$ is de-noised signal.

In the threshold de-noising, a new method of threshold function is proposed according to the characteristics of hard and soft:

$$
\begin{aligned}
& s= \begin{cases}x & |x| \geq t \\
\operatorname{sign}(|x|) \cdot \varphi(x) / t^{4} & \frac{t}{2} \leq|x|<t \\
0 & |x| \leq \frac{t}{2}\end{cases} \\
& \varphi(x)=x^{5} /(x-t)^{2}
\end{aligned}
$$

\section{Experiment and result analysis}

The proposed improved threshold denoising algorithm based on EMD is used to denoise the eddy current array amplitude ratio signal of channel first, and the SNR of channel first is $10.5120 \mathrm{~dB}$.

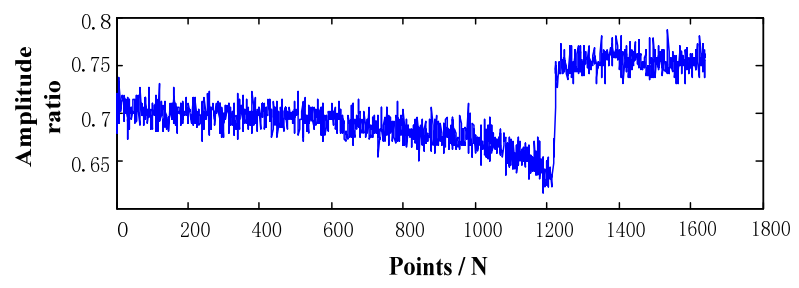

a. signal with noise

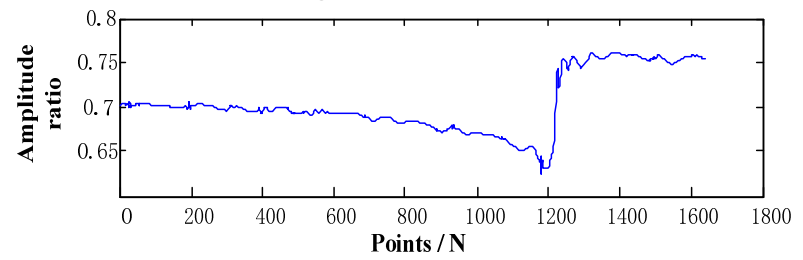

c. signal after EMD threshold de-noising

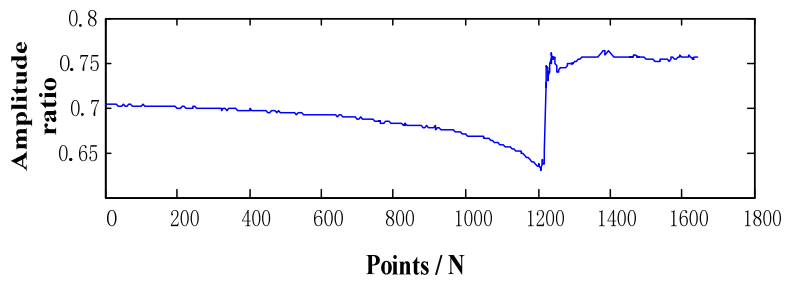

b. non-noise signal

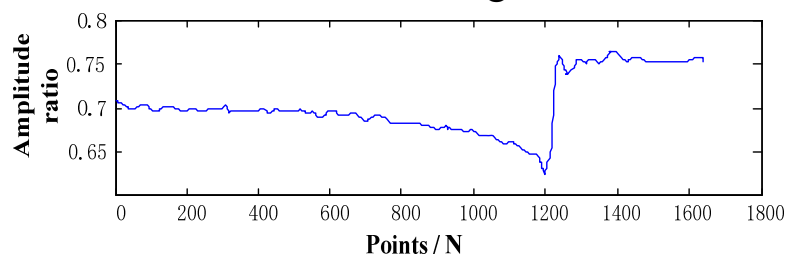

d. signal after new EMD threshold de-noising

Fig. 1 Two methods of de-noising results figure contrast in channel first

Figure 1shows that the EMD threshold denoising causes the signal distortion; the new EMD threshold improved algorithm can reduce the distortion, better recovery signal. In order to quantitatively analyze the effect of noise elimination, the two parameters are set as follows:

(1) SNR(signal to noise ratio):

$$
S N R=10 \log \left[\frac{\sum_{i=1}^{n}\left|x_{i}\right|^{2}}{\sum_{i=1}^{n}\left|\bar{x}_{i}-x_{i}\right|^{2}}\right]
$$

(2) RMSE(Root mean square error):

$$
R M S E=\sqrt{\frac{1}{n} \sum_{i=1}^{n}\left(x_{i}-\bar{x}_{i}\right)^{2}}
$$

Comparison of different methods is as follows:

Table 1 Two methods comparing in channel first

\begin{tabular}{cccc}
\hline Numble & $\begin{array}{c}\text { signal with } \\
\text { noise }\end{array}$ & $\begin{array}{c}\text { EMD threshold } \\
\text { denoising }\end{array}$ & $\begin{array}{c}\text { improved EMD } \\
\text { threshold denoising }\end{array}$ \\
\hline SNR & 10.5120 & 18.3723 & 19.9021 \\
RMSE & 0.0062 & 0.0025 & 0.0023 \\
\hline
\end{tabular}


By comparing the data of Table 1, we can know that the improved EMD threshold denoising algorithm has improved the SNR, reduce the RMSE, and be more effective to recover the original signal.

The improved EMD threshold denoising is used to eliminate the noise of the five channel amplitude ratio signals, and the denoising effect is judged by SNR. The SNR after de-noising is shown in Figure 2:

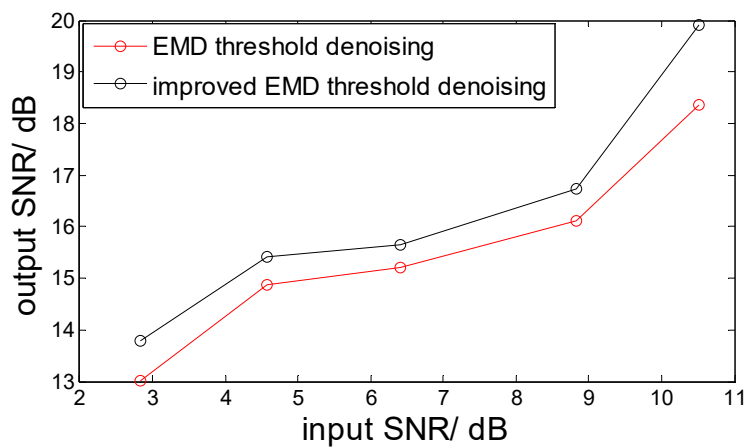

Fig. 2 Comparison of different methods for SNR

Figure 2 shows that for different channel signals, the improved EMD threshold algorithm can better denoise the noise than the old algorithm. Especially when signal has much noise, the improved EMD threshold algorithm can significantly improve the SNR, showing a good performance of denoising.

\section{Conclusion}

After the theoretical analysis and the numerical experiment, we can draw a conclusion that the improved EMD threshold algorithm can better denoise the noise in eddy current array signal and be more effective to recover the original signal than the original EMD threshold algorithm.

\section{Acknowledgments}

The study is supported by the National Nature Science Foundation of China (No. 51507186). This support is gratefully acknowledged.

\section{References}

[1] Nihtianov S. R.A. Measuring in the Subnanometer Range: Capacitive and Eddy Current Nanodisplacement Sensors. IEEE Industrial Electronics Magazine. 8 (2014) 6-15.

[2] Takano C, Ohta Y. Heart rate measurement based on a time-lapse image. Medical Engineering \& Physics. 29(2007)853-857.

[3] Huang N E, Shen Z, Long S R, et al. The empirical mode decomposition and the Hilbert spectrum for nonlinear and non-stationary time series analysis. Proceedings of the Royal Society of London, Series A, Mathematical, Physical and Engineering Sciences. 454 (1998) 903-995.

[4] Ning B, Qiyu S, Zhi hua Y, et al. Robust image watermarking based on multiband wavelets and empirical mode decomposition. IEEE Trans on Image Process. 8(2007)1956-1966.

[5] Kopsinis Y, McLauglin S. Development of EMD-Based Denoising Methods Inspired by Wavelet Thresholding. IEEE Transactions on Signal Processing. 57(2009) 1351-1362.

[6] MONTILLET J.-P, TREGONING P, MCCLUSKY S, et al. Extracting white noise statistics in GPS coordinate time series. IEEE Geoscience and Remote Eensing Letters. 10(2013): 563-567. 